\title{
Maternal high-fat diet alters expression of pathways of growth, blood supply and arachidonic acid in rat placenta
}

\author{
Marloes Dekker Nitert ${ }^{1}{ }^{*},{\text { Kanchan } \text { Vaswani }^{1} \text {, Melissa Hum }}^{1}$, Hsiu-Wen Chan ${ }^{1}$, Ryan Wood-Bradley ${ }^{2}$, \\ Sarah Henry ${ }^{2}$, James A. Armitage ${ }^{2,3}$, Murray D. Mitchell ${ }^{1}$ and Gregory E. Rice ${ }^{1}$ \\ ${ }^{1}$ University of Queensland Centre for Clinical Research, Royal Brisbane and Women's Hospital Campus, Herston, QLD, Australia 4029 \\ ${ }^{2}$ Department of Anatomy and Developmental Biology, Monash University, Clayton, VTC, Australia 3800 \\ ${ }^{3}$ School of Medicine (Optometry), Faculty of Health, Deakin University, Waurn Ponds, VIC, Australia
}

(Received 23 May 2013 - Final revision received 10 September 2013 - Accepted 20 September 2013)

Journal of Nutritional Science (2013), vol. 2, e41, page 1 of 7

doi:10.1017/jns.2013.36

Abstract

The high fat content in Western diets probably affects placental function during pregnancy with potential consequences for the offspring in the short and long term. The aim of the present study was to compare genome-wide placental gene expression between rat dams fed a high-fat diet (HFD) and those fed a control diet for 3 weeks before conception and during gestation. Gene expression was measured by microarray and pathway analysis was performed. Gene expression differences were replicated by real-time PCR and protein expression was assessed by Western blot analysis. Placental and fetal weights at E17.25 were not altered by exposure to the maternal HFD. Gene pathways targeting placental growth, blood supply and chemokine signalling were upregulated in the placentae of dams fed the HFD. The up-regulation in messenger RNA expression for five genes Ptgs2 (fatty acid cyclo-oxidase 2; COX2), Limk1 (LIM domain kinase 1), Pla2g2a (phospholipase A2), Itga1 (integrin $\alpha$-1) and Serpine1 was confirmed by real-time PCR. Placental protein expression for COX2 and LIMK was also increased in HFD-fed dams. In conclusion, maternal HFD feeding alters placental gene expression patterns of placental growth and blood supply and specifically increases the expression of genes involved in arachidonic acid and PG metabolism. These changes indicate a placental response to the altered maternal metabolic environment.

Key words: Placenta: High-fat diet: Arachidonic acid: LIM domain kinase 1

The highly vascularised placenta supplies nutrients to the developing fetus and epidemiological studies have shown that impaired fetal growth and the development of adult-onset disease are strongly associated with compromised placental structure and function. Converging lines of evidence now suggest that the placenta plays a significant role in intra-uterine programming by conveying critical growth-regulating signals between mother and fetus. Maternal overnutrition, which is increasingly common in the developed world, has been reported to alter placental function and structure and thereby the fetal growth trajectory $^{(1)}$. In C57BL/ 6 mice, a high-fat diet (HFD) has been found to induce placental oxidative stress, causing vascular dysfunction and thereby disrupted nutrient transport ${ }^{(1,2)}$. Similar findings of altered vascular development have been reported in the placentae of Sprague-Dawley rats fed a $\mathrm{HFD}^{(3)}$. In Japanese macaques maintained on a chronic HFD, placental expression of a range of cytokines and Toll-like receptor 4 (TLR-4) is increased when compared with animals maintained on a normal diet ${ }^{(4)}$. Gene expression of specific targets in the placenta has been studied previously. Recently, it was reported that messenger RNA (mRNA) expression of GLUT1 (S/c2a1) is down-regulated whereas that of GLUT3 (S/c2a3) is up-regulated in placentae from HFD-fed rats ${ }^{(5)}$. Similarly, up-regulation of mRNA expression of the cationic amino acid transporter Slc7a1 and

Abbreviations: COX2, cyclo-oxidase 2; HFD, high-fat diet; LIMK1, LIM domain kinase 1; mRNA, messenger RNA

* Corresponding author: Dr Marloes Dekker Nitert, fax +61 73346 5509, email m.dekker@uq.edu.au 
down-regulation of the Na-dependent amino acid transporter Slc38a4 is observed in the placentae from HFD-fed rats ${ }^{(5)}$. The mechanisms underlying the changes in placental morphology and gene expression are incompletely described. It is known, however, that HFD feeding increases the expression of imprinted genes such as the $\operatorname{Ig} 2 \mathrm{~g}$ gene ${ }^{(6)}$. This indicates decreased levels of methylation which may be secondary to the reported decreased expression levels of the DNA methyltransferases Dnmt1 and Dnmt3a ${ }^{(5)}$.

In mice, a HFD exerts distinct effects on genome-wide placental gene expression ${ }^{(6-8)}$. Mao et al. reported that both a HFD and a low-fat diet have pronounced and specific effects on placental gene expression that are different for male and female fetuses, with larger changes observed in females ${ }^{(7)}$. Sexual dimorphic patterns were similarly observed in the expression and DNA methylation levels of imprinted genes in the placenta of another mouse model on a HFD ${ }^{(\sigma)}$. When genome-wide gene expression was studied in this last model, the HFD altered the placental gene expression of both female and male fetuses but only a fraction of the genes overlapped between the sexes. While there have been reports on the effects of HFD feeding on mRNA expression of specific placental genes, there are no studies on the effects of maternal HFD feeding on global placental gene expression in the rat. The aim of the present study, therefore, was to characterise genome-wide placental gene expression to identify genes and pathways commonly affected by HFD feeding in male and female rat fetuses.

\section{Materials and methods}

\section{Animals}

Female Sprague-Dawley rats, aged 8-9 weeks, were obtained and allowed to acclimatise for 1 week before diet onset. The animals were maintained in a light-controlled environment $(12 \mathrm{~h}$ light $-12 \mathrm{~h}$ dark cycle; $24^{\circ} \mathrm{C}$ ) throughout the study. After 1 week, female rats were randomly allocated to a hyperenergetic HFD (SF08-023; Specialty Feeds) or a control diet (SF09-091) (Table 1). The fat component of the HFD consisted of pork lard and rapeseed oil; in the control diet the fat component was rapeseed oil only. Both diets contained sucrose, wheat starch and dextrinised starch as sources of carbohydrates, although to different extents. The diets had similar contents of vitamins and minerals. After 3 weeks, the female rats were timemated for $3 \mathrm{~h}$ with male Sprague-Dawley rats fed a control diet. This day was designated as embryonic day zero (E0). After mating, the dams were individually housed and maintained on their respective diets, having food and water ad libitum until killing at E17.25, a stage in pregnancy in which there is rapid fetal growth. Placentae were obtained and weighed, snap-frozen in liquid $\mathrm{N}_{2}$ and stored at $-80^{\circ} \mathrm{C}$. Approval was obtained from the School of Biomedical Sciences Animal Ethics Committee at Monash University (SOBSA/2008/39).

\section{Gene expression microarray}

A quantity of $30 \mathrm{mg}$ placental tissue (wet weight) from one placenta per dam on the HFD $(n 4)$ or the control diet $(n 6)$
Table 1. Diet composition

\begin{tabular}{lcc}
\hline & Control diet & High-fat diet \\
\hline Protein (\%) & 19.4 & 23.3 \\
Total fat (\%) & 7.0 & 23.5 \\
Carbohydrates (\%) & 73.6 & 53.2 \\
Digestible energy (MJ/kg) & 16.1 & 19.1 \\
Energy from protein (\%) & 21.0 & 21.0 \\
Energy from lipids (\%) & 16.0 & 42.2 \\
Energy from carbohydrates (\%) & 63.0 & 36.8 \\
Fatty acid composition & & \\
$16: 0$ (\%) & 0.40 & 5.60 \\
$18: 0(\%)$ & 0.10 & 0.10 \\
$18: 1$ (\%) & 4.20 & 8.60 \\
$18: 2(\%)$ & 1.30 & 3.60 \\
$18: 3(\%)$ & 0.98 & 0.50 \\
Total $n$-3 (\%) & 0.98 & 0.73 \\
Total $n-6(\%)$ & 1.51 & 3.63 \\
Total MUFA (\%) & 3.98 & 9.22 \\
Total PUFA (\%) & 2.50 & 4.47 \\
Total SFA (\%) & 0.50 & 9.83 \\
\hline
\end{tabular}

was homogenised with a mortar and pestle in liquid $\mathrm{N}_{2}$. RNA was isolated with the AllPrep DNA/RNA mini kit (Qiagen) according to the manufacturer's specifications. Total RNA was quantified and its quality assessed on a Bioanalyser (Agilent 2100). RNA samples with RNA integrity number $>7,260: 280$ ratio $>2$ and 260:230 ratio $>1$ were selected for microarray analysis.

Total RNA (500 ng) was converted and biotinylated with the Illumina TotalPrep RNA amplification kit (Illumina). Biotinylated samples were hybridised to the RatRef- 12 Expression BeadChip (Illumina), incorporating twelve samples per chip. The chip was scanned on a BeadStation 500 System using Beadscan software v3.5.31. Gene expression data were normalised by probe-intensity transformation and normalisation (Lumi package for the analysis of Illumina microarray data in the bioconductor software suite $\left.{ }^{(9)}\right)$. Differences in expression between the HFD and control diets were calculated with the unpaired Student's $t$ test, with $P<0.05$ considered to be statistically significant. Pathway analysis on up- or downregulated genes with a $P<0.05$ was based on GO/KEGG by the Web-based Gene Set Analysis Toolkit (Webgestalt; http://bioinfo.vanderbilt.edu/webgestalt). A further pathway analysis was undertaken on a list of genes with a fold-change of 1.4 for up-regulated genes. Both pathway analyses were repeated with the DAVID algorithm (http://david.abcc. ncifcrf.gov) to ensure the validity of the pathway analyses.

\section{Quantitative real-time PCR}

Validation of the microarray results for six genes with high fold-changes was performed by real-time PCR. RNA isolated from ten individual HFD placentae and ten individual control placentae (one placenta per dam) that included all the samples analysed by microarray was reverse transcribed into complementary DNA (QuantiTect cDNA synthesis kit; Qiagen). A total of six genes were selected for validation: five genes that were up-regulated (Ptgs2, Pla2g2a, Serpine1, Itga1 and Limk1; encoding for cyclo-oxidase 2, phospholipase A2, 
Serpin1, integrin $\alpha-1$ and LIM domain kinase 1, respectively) and one gene that was down-regulated (Umps; encoding for UMP synthetase). The up-regulated genes had a fold-change of $>1.4$ and $P<0.05$. The down-regulated gene had a foldchange of $<0.71$ and $P<0 \cdot 05$. SYBR green assays were designed on the National Center for Biotechnology Information (NCBI) primer design tool. All assays spanned exon-exon junctions and primer specificity was verified by PrimerBlast (NCBI). Primer sequences can be found in Supplementary Table 1. Primer efficiency was established and dissociation curve analysis confirmed the presence of one PCR product at the expected melting temperature. Gene expression for $\beta$-actin (Actb) was used as the endogenous control. Real-time PCR was performed in a $20 \mu \mathrm{l}$ volume with $10 \mu \mathrm{l}$ SYBR green PCR master mix (Life Technologies), 20 ng complementary DNA and 300 nm forward and reverse primers, respectively in the StepOnePlus real-time PCR system (Life Technologies). Gene expression was compared with the comparative cycle threshold $\left(\mathrm{C}_{\mathrm{T}}\right)$ method $\left(2_{\mathrm{T}}{ }^{-\Delta \Delta \mathrm{C}}\right)$.

\section{Western blot}

Placental tissue $(50 \mathrm{mg})$ was homogenised with a mortar and pestle, and lysed in ProteoJET Mammalian Cell Lysis Reagent with protease inhibitors (PhosSTOP Phosphatase Inhibitor Cocktail; Roche Applied Science). Protein concentration was determined by the bicinchoninic acid method with bovine serum albumin as the protein standard (Pierce, ThermoFisher Scientific). A quantity of $30 \mu \mathrm{g}$ of total protein was loaded onto NuPAGE Bis-Tris Gel (4-12 or 12\%) (Life Technologies). Proteins were transferred onto PVDF (polyvinylidene fluoride) membranes (Millipore). The membrane was blocked in $5 \%$ skimmed milk in PBS-Tween for $1 \mathrm{~h}$ at room temperature before overnight incubation with primary antibody for fatty acid cyclo-oxidase 2 (COX2) (sc-7951; Santa Cruz) and $\beta$-actin (A5316, SigmaAldrich) at $4^{\circ} \mathrm{C}$. Secondary horseradish peroxidase (HRP)-conjugated antibodies (donkey anti rabbit HRP, Sigma 90545 and donkey anti mouse HRP, Santa Cruz Biotechnology 2314) were incubated for $1 \mathrm{~h}$ at room temperature in a Super Signal West Dura, extended duration (Pierce 34075) and the blots were developed for $5 \mathrm{~min}$ before scanning on the GS0800 imaging system (BioRad). For LIMK1, rabbit primary antibody (ab81046; Abcam) Secondary LI-COR ${ }^{\circledR}$ antibody (goat anti-rabbit $800 \mathrm{CW}$ LI-COR ${ }^{\circledR}$ 926-32211 and donkey anti-mouse 680LT LI-COR ${ }^{\circledR}$ 926-68022) was incubated for $1 \mathrm{~h}$ at room temperature and protein was detected by the Odyssey Infrared Imaging System (LI-COR Biosciences). Protein expression was analysed by densitometry, with $\beta$-actin used to normalise for differences in protein loading.

\section{Statistical analysis}

Individual placentae were investigated for all experiments, and each placenta was obtained from an independent litter. For all analyses, between-group comparisons of the means were made with unpaired Student's $t$ test after confirming that the data were normally distributed. The microarray analyses were performed on six placentae from dams on the control diet and four placentae from dams on the HFD. The quantitative PCR replication experiments were carried out on RNA isolated from ten placentae per group. The Western blot analyses were performed on protein from ten placentae each for COX2, and on protein from nine placentae from the control diet group and six HFD placentae for LIMK1. Data were presented as mean values with their standard errors; $P<0.05$ was considered to be statistically significant.

\section{Results}

\section{Fetal and placental weights}

Fetal and placental weights were similar in the control and HFD groups at E17.25.

Fetal weights were similar in rats on the control diet $(0.69$ (SEM 0.02) g) and the HFD (0.68 (SEM 0.03) g; $P=0.87$ ) as were placental weights (control diet 0.36 (SEM 0.02) v. HFD 0.35 (SEM 0.02) g; $P=0 \cdot 91$ ). Maternal weights were not different at E17.25 between the diet groups (control diet 328.5 (seM 33.4) $v$. HFD 347.5 (sem 33.3) g; $P=0.69$ ).

\section{Genome-wide gene expression}

Microarray analysis of placental RNA from six dams on the control diet and four dams on the HFD yielded 825 differentially expressed genes with a cut-off of $P<0.05$; none of the genes were differentially expressed after correction for multiple testing. Of the 825 genes, there were 457 annotated genes with 327 up-regulated and 130 down-regulated genes in the placentae of dams on the HFD (Supplementary Table 2). Pathway analyses were performed separately for up-regulated and down-regulated genes on the basis of the KEGG/GO database with two different algorithms, with the top ten pathways for each presented in Table 2.

Pathway analysis was also performed on nineteen up-regulated genes with a fold-change larger than 1.4 in HFD-fed dams (Supplementary Table 3). The pathway analysis was based on the gene ontology database and seven pathways were found with the Webgestalt tool (Table 3); all of these were repeated with the DAVID pathway analysis tool. The main pathways included regulation of apoptosis, growth regulation, circulation and chemotaxis.

The microarray results were confirmed by real-time PCR for five up-regulated genes and one down-regulated gene with high fold-changes: Limk1, Itga1, Pla2g2a, Ptgs2, Serpine1 and Umps. These genes were selected based on their known functions and their prominence in the pathway analyses. Differential expression was observed for the genes that were up-regulated in placentae from HFD-fed dams, for example, Limk1 (foldchange 4.1), Itga1 (fold-change 1.8), Pla2g2a (fold-change 1.9), Ptgs2 (fold-change 1.9), Serpine1 (fold-change 2.0) but not for the down-regulated gene Umps (fold-change 1.7) (Fig. 1). We investigated if the change in mRNA expression translated to a change in protein expression for fatty acid COX2 (EC 1.44.99.1), which is encoded by Ptgs2, and the serine/threonine kinase LIMK1, which is encoded by Limk1. 
Table 2. Pathway analysis of all up-regulated and down-regulated genes with $P<0.05$ with the Webgestalt pathway analysis tool

\begin{tabular}{|c|c|c|c|c|c|}
\hline Pathway (total number of genes in pathway) & $\mathrm{O}$ & E & $\mathrm{R}$ & $P$ & Adjusted $P$ \\
\hline \multicolumn{6}{|l|}{ Up-regulated genes } \\
\hline Metabolic pathways $(1169)^{*}$ & 28 & 8.09 & 3.46 & $1.6 \times 10^{-8}$ & $1.34 \times 10^{-6}$ \\
\hline MAPK signalling (269)* & 10 & 1.76 & 5.37 & $2.07 \times 10^{-5}$ & 0.0009 \\
\hline $1 \mathrm{C}$ pool by folate $(18)^{*}$ & 3 & 0.12 & $24 \cdot 10$ & 0.0002 & 0.0028 \\
\hline Lysosome $(124)^{\star}$ & 6 & 0.86 & 7.00 & 0.0002 & 0.0028 \\
\hline Antigen processing and presentation $(91)^{*}$ & 5 & 0.63 & 7.94 & 0.0004 & 0.0048 \\
\hline Alzheimer's disease (212) & 8 & 1.47 & 5.46 & 0.0001 & 0.0021 \\
\hline Apoptosis $(93)^{*}$ & 4 & 0.64 & $6 \cdot 22$ & 0.0040 & 0.026 \\
\hline Glycosphingolipid biosynthesis - ganglio series $(15)^{\star}$ & 2 & 0.10 & $19 \cdot 28$ & 0.0047 & 0.026 \\
\hline Chemokine signalling (178) & 5 & 1.23 & 4.06 & 0.0082 & 0.035 \\
\hline Arachidonic acid metabolism (74) & 3 & 0.51 & 5.86 & 0.0148 & 0.042 \\
\hline \multicolumn{6}{|l|}{ Down-regulated genes } \\
\hline Alzheimer's disease $(212)^{*}$ & 5 & 0.56 & 8.99 & 0.0003 & 0.0075 \\
\hline RNA degradation $(77)^{*}$ & 3 & 0.20 & 14.85 & 0.0011 & 0.0081 \\
\hline Peroxisome $(80)^{\star}$ & 3 & 0.21 & $14 \cdot 29$ & 0.0012 & 0.0081 \\
\hline Proximal tubule bicarbonate reclamation (20) & 2 & 0.05 & $38 \cdot 11$ & 0.0013 & 0.0081 \\
\hline Metabolic pathways (1169)* & 9 & 3.07 & 2.93 & 0.0038 & 0.016 \\
\hline Leucocyte transendothelial migration (114) & 3 & 0.30 & $10 \cdot 03$ & 0.0034 & 0.016 \\
\hline Tight junction $(131)^{\star}$ & 3 & 0.34 & 8.73 & 0.0061 & 0.018 \\
\hline Notch signalling (50) & 2 & 0.13 & $15 \cdot 24$ & 0.0077 & 0.024 \\
\hline Basal cell carcinoma (53) & 2 & 0.14 & 14.38 & 0.0086 & 0.024 \\
\hline Pathways in cancer (319) & 4 & 0.84 & 4.78 & 0.0101 & 0.025 \\
\hline
\end{tabular}

O, Observed number of genes; E, expected number of genes; R, O:E ratio; MAPK, mitogen-activated protein kinase.

* Pathways also found with the DAVID pathway analysis algorithm.

\section{Protein expression}

Compared with controls, placentae from dams fed the HFD showed a 1.3 -fold increase in protein expression of COX2 (control diet 0.64 (SEM 0.050) $v$. HFD $0 \cdot 81$ (SEM 0.051); $P<0.05)$ in line with the increased mRNA expression (Fig. 2(a)). Similarly, LIMK1 was found to have increased protein expression by 1.4 -fold in placentae from HFD-fed dams (control diet 0.16 (SEM 0.017) $v$. HFD 0.22 (SEM 0.009); $P<0 \cdot 05$ ) (Fig. 2(b)).

\section{Discussion}

The aim of the present study was to establish the effects of a maternal HFD on rat placental gene expression using a genome-wide approach. The HFD was associated with the differential expression of more than 800 placental probes, corresponding to 457 known genes. Many of the up-regulated genes in the HFD group were associated with signal transduction pathways, including those involving phospholipid-derived mediators, cytokines and chemokines. This is not unexpected given the known associations of HFD feeding with low-grade inflammation ${ }^{(10,11)}$. Metabolic pathways in the placenta were also significantly affected by the HFD. Regulation of metabolic pathways in the placenta by overnutrition and undernutrition has been reported previously in mouse models ${ }^{(7,12)}$, specifically, placental expression differences between male and female offspring of dams on a control diet for amino acid and carbohydrate metabolism pathways and for lipid metabolism in HFD-fed dams but not in metabolism pathways

Table 3. Pathway analysis based on gene ontology in the Webgestalt pathway analysis tool with eighteen up-regulated genes with a fold-change $>1.4$ or down-regulated with a fold-change $<0.7$

\begin{tabular}{|c|c|c|c|c|c|}
\hline Pathway (total number of genes in pathway) & $\mathrm{O}$ & E & $\mathrm{R}$ & $P$ & Adjusted $P$ \\
\hline $\begin{array}{l}\text { Regulation of apoptosis (729) } \\
\text { Includes Ptgs2 and Itga1 }\end{array}$ & 5 & 0.97 & $5 \cdot 15$ & 0.0021 & 0.044 \\
\hline $\begin{array}{l}\text { Regulation of angiogenesis (59) } \\
\text { Includes Serpine1 }\end{array}$ & 2 & 0.08 & $25 \cdot 47$ & 0.0027 & 0.044 \\
\hline $\begin{array}{l}\text { Cell chemotaxis }(47) \\
\text { Includes Itga1 }\end{array}$ & 2 & 0.06 & 31.97 & 0.0017 & 0.044 \\
\hline $\begin{array}{l}\text { Regulation of anatomical structure size (347) } \\
\text { Includes Ptgs2, Limk1 and Itga1 }\end{array}$ & 4 & 0.46 & 8.66 & 0.001 & 0.044 \\
\hline $\begin{array}{l}\text { Developmental growth (173) } \\
\text { Includes Ptgs2, Limk1 and Serpine2 }\end{array}$ & 3 & 0.23 & 13.03 & 0.0015 & 0.044 \\
\hline $\begin{array}{l}\text { Blood circulation (204) } \\
\text { Includes Ptgs2 and Itga1 }\end{array}$ & 3 & 0.27 & 5.09 & 0.0023 & 0.044 \\
\hline $\begin{array}{l}\text { Oxidoreductase activity, acting on single donors with } \\
\text { incorporation of molecular oxygen (38) } \\
\text { Includes Ptgs2 }\end{array}$ & 2 & 0.04 & $47 \cdot 65$ & 0.0008 & 0.034 \\
\hline
\end{tabular}

$\mathrm{O}$, Observed number of genes; $\mathrm{E}$, expected number of genes; $\mathrm{R}, \mathrm{O}: \mathrm{E}$ ratio. 

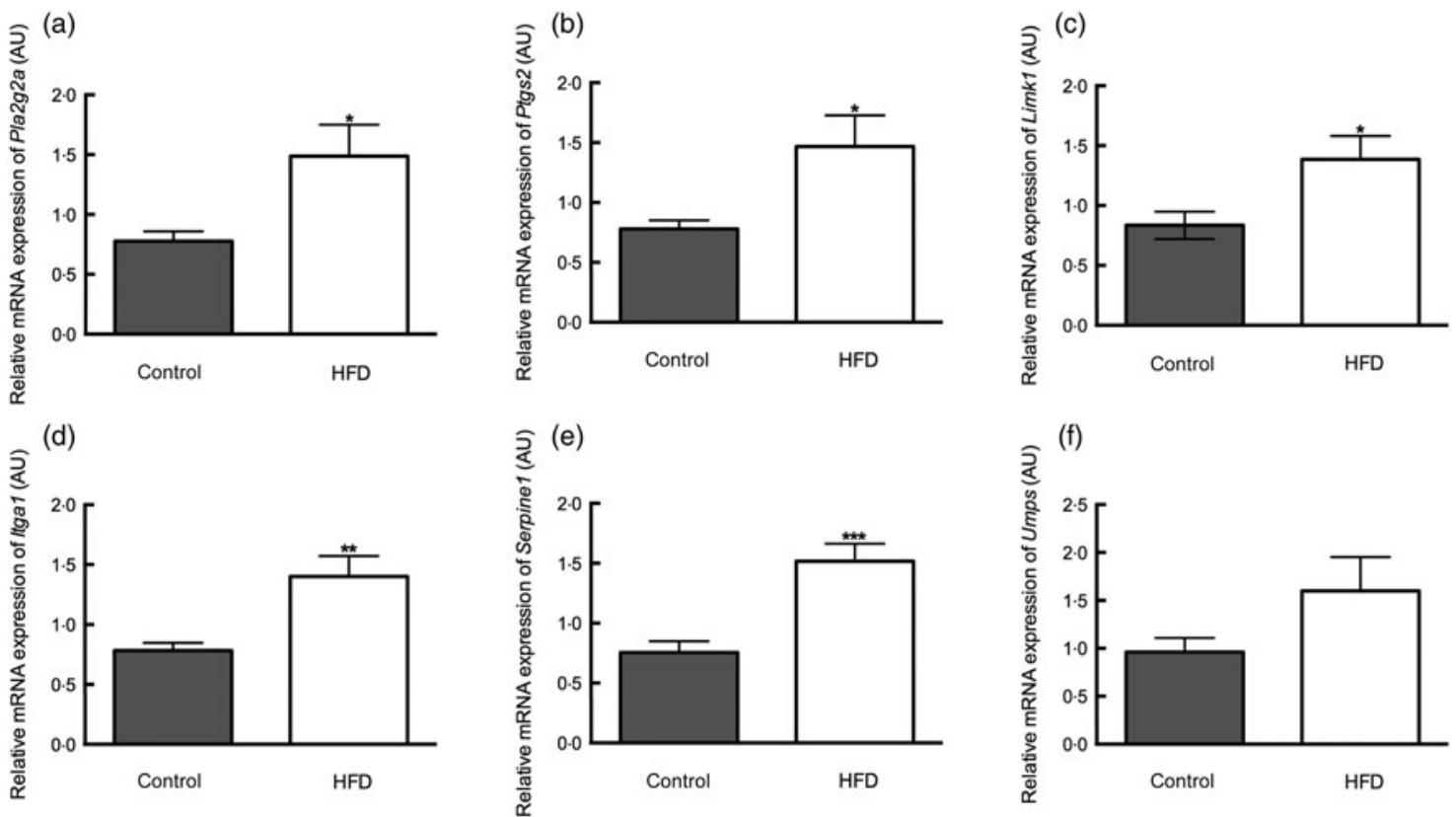

Fig. 1. Replication of microarray results with real-time PCR for Pla2g2a, encoding for phospholipase A2 (a), Ptgs2, encoding for cyclo-oxidase 2 (b), Limk1, encoding for LIM domain kinase 1 (c), Itga1, encoding for integrin $\alpha-1$ (d), Serpine1, encoding for Serpin1 (e) and Umps, encoding for UMP synthetase (f). mRNA, messenger RNA; AU, arbitrary units; HFD, high-fat diet. Values are means ( $n 10$ placentae (one placenta per dam) in each group), with standard errors represented by vertical bars. Mean value was significantly different from that of the control group: ${ }^{\star} P<0.05,{ }^{* \star} P<0.01,{ }^{* \star} P<0.001$.
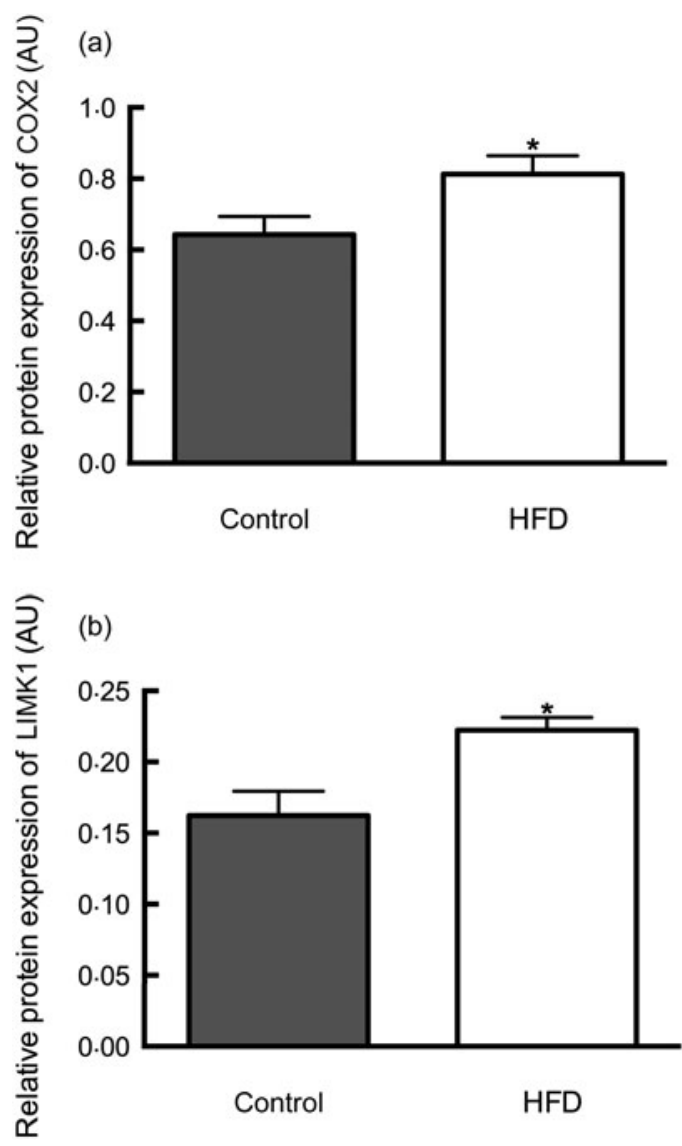

Fig. 2. Placental protein expression of cyclo-oxidase 2 (COX2) (a) and LIM domain kinase 1 (LIMK1) (b). AU, arbitrary units; HFD, high-fat diet. Values are means ( $n 10$ placentae (one placenta per dam) in each group for COX2; $n 9$ in control group and $n 6$ in HFD group for LIMK1), with standard errors represented by vertical bars. *Mean value was significantly different from that of the control group $(P<0.05)$. between the diets ${ }^{(8)}$. In addition, pathway analyses indicated that methylation may be altered by HFD feeding since mRNA expression of genes in the $1 \mathrm{C}$ pool by folate pathway was significantly altered. This is in accordance with the results obtained by Lin et al. ${ }^{(5)}$ who reported that expression levels of the DNA methyltransferases Dnmt1 and Dnmt3a were decreased in the placenta of rats fed a HFD. In female offspring of mouse dams fed a HFD, decreased expression of Dnmt3l has been reported ${ }^{(8)}$. Decreased levels of methylation could result in increased levels of gene expression. In the present study, the HFD resulted in increased gene expression of more genes than the number of genes with decreased levels of gene expression.

The pathway analysis conducted including only the nineteen up-regulated genes with a fold-change of $>1.4$ indicated alterations in pathways that modulate angiogenesis and circulating blood as well as growth and development. Heightened expression of genes in these pathways could lead to increased growth of the placenta and thereby the fetus. HFD feeding in mice has been reported to increase offspring neonatal fat mass ${ }^{(13)}$. At E17.25 there were no differences in either placental or fetal weight, and although fetal weight increased by approximately $2.3 \mathrm{~g}$ between E17.25 and birth there were no effects on fetal weight caused by the maternal HFD (data not shown).

The pathway analysis of down-regulated genes yielded three pathways common to both pathway analyses: Alzheimer's disease, RNA metabolism and cell adhesion. The Alzheimer's disease pathway encompasses many cellular processes including receptor signalling, oxidative phosphorylation and $\mathrm{Ca}$ signalling, which all lead to apoptosis. Alzheimer's disease has been associated with the metabolic syndrome in humans ${ }^{(14)}$ 
and Wistar Kyoto rats fed a HFD show increases in the levels of biomarkers for Alzheimer's disease ${ }^{(15)}$. Apoptosis was one of the main pathways that we reported for the analysis with all genes that were differentially expressed in the placentae of dams on the HFD or the control diet. Decreases in this pathway could result in reduced levels of apoptosis and could thereby also lead to increased placental and fetal size. Indeed, increased levels of placental apoptosis have previously been correlated to reduced placental size in primate and rodent models of maternal undernutrition ${ }^{(12,16,17)}$.

The arachidonic acid metabolism pathway was also significantly altered in the placentae of rats fed the HFD. COX2 belongs to this pathway and its mRNA and protein expression was increased in placentae from HFD-fed rat dams. mRNA for Ptgs2, Ptgs1 and the PG-metabolising enzyme Hpgd was increased in the placentae of female but not male offspring of HFD-fed mouse dams at E12.5 ${ }^{(7)}$. In humans, increased placental mRNA expression of PTGS2 has been reported in pregnancies complicated with pre-eclampsia in some ${ }^{(18,19)}$ but not all studies ${ }^{(20,21)}$. This increase in expression was localised to the syncytiotrophoblast cells ${ }^{(18)}$. In pre-eclampsia, the vascularisation of the placenta is dysregulated ${ }^{(22,23)}$. Fetal polymorphisms in PTGS2 have been shown to cause placental malperfusion in 132 twin pregnancies ${ }^{(24)}$, again indicating a role for COX2 in placental blood flow. The mRNA expression of Pla2g2a, which encodes for phospholipase A2, the enzyme catalysing the reaction from phosphatidylcholine to arachidonic acid and lysophosphatidylcholine, was also increased, providing substrate for COX2. A high gene expression of phospholipase A2 was also reported in placentae from obese neonates and was correlated to accumulation of $n-3$ fatty acids ${ }^{(25)}$. In the present study, neonatal circulating $n$-3 fatty acids and neonatal body composition were not measured.

Expression for LIMK1 showed the largest difference in expression between the control diet and the HFD. LIMK1 encodes a serine/threonine protein kinase which contains two LIM domains. It is a modulator of actin and microtubule dynamics $^{(26)}$ and has been implicated in the mitotic process ${ }^{(27)}$. In the mouse, LIMK1 has been shown to be expressed early in the embryo as well as in trophoblast giant cells ${ }^{(28)}$.

A limitation of the present study is the relatively small sample size. This precluded an analysis of the effects of sex in the response to HFD feeding. In mice, genome-wide placental gene expression has been carried out and analysis has shown that HFD feeding resulted in differential gene expression of many genes in a sexually dimorphic pattern, with females showing larger changes than males ${ }^{(6-8)}$. In these studies, a slightly larger number of placentae or pools of placentae were studied for each condition and results were not corrected for multiple testing either. However, the present study found changes in similar genes ${ }^{(7)}$ and similar pathways ${ }^{(8)}$ to the studies in mice, indicating common effects of a HFD on placental gene expression.

\section{Conclusion}

Maternal HFD feeding alters placental gene expression at E17.25, with pathways promoting inflammation and growth being over-represented among up-regulated genes and those affecting apoptosis among down-regulated genes. COX2, phospholipase A2 and LIMK1 mRNA and protein expression were up-regulated after maternal HFD feeding, implicating arachidonic acid and PG metabolism as targets of maternal diet effects.

\section{Supplementary material}

To view supplementary material for this article, please visit http://dx.doi.org/10.1017/jns.2013.36

\section{Acknowledgements}

The present study was partially supported by a University of Queensland grant (to M. D. M.). M. D. N. is supported by the Patricia Dukes Postdoctoral Fellowship from the Royal Brisbane and Women's Hospital Foundation. J. A. A. is supported by a fellowship of the National Heart Foundation. G. E. R. was in receipt of an Australian Government National Health and Medical Research Council (NHMRC) Principal Research Fellowship.

M. D. N. performed the analysis and wrote the manuscript. K. V., M. H., H.-W. C., R. W.-B. and S. H. collected data, performed analyses and reviewed the manuscript. J. A. A., M. D. M. and G. E. R. conceived of the study and reviewed the manuscript.

There are no conflicts of interest.

\section{References}

1. Jones HN, Woollett LA, Barbour N, et al. (2009) High-fat diet before and during pregnancy causes marked up-regulation of placental nutrient transport and fetal overgrowth in C57/BL6 mice. FASEB J 23, 271-278.

2. Liang C, DeCourcy K \& Prater MR (2010) High-saturated-fat diet induces gestational diabetes and placental vasculopathy in C57BL/ 6 mice. Metabolism 59, 943-950.

3. Hayes EK, Lechowicz A, Petrik JJ, et al. (2012) Adverse fetal and neonatal outcomes associated with a life-long high fat diet: role of altered development of the placental vasculature. PLOS ONE 7, e33370.

4. Frias AE, Morgan TK, Evans AE, et al. (2011) Maternal high-fat diet disturbs uteroplacental hemodynamics and increases the frequency of stillbirth in a nonhuman primate model of excess nutrition. Endocrinology 152, 2456-2464.

5. Lin Y, Zhuo Y, Fang ZF, et al. (2012) Effect of maternal dietary energy types on placenta nutrient transporter gene expressions and intrauterine fetal growth in rats. Nutrition 28, 1037-1043.

6. Gallou-Kabani C, Gabory A, Tost J, et al. (2010) Sex- and dietspecific changes of imprinted gene expression and DNA methylation in mouse placenta under a high-fat diet. PLOS ONE 5, e14398.

7. Mao J, Zhang X, Sieli PT, et al. (2010) Contrasting effects of different maternal diets on sexually dimorphic gene expression in the murine placenta. Proc Natl Acad Sci U S A 107, 5557-5562.

8. Gabory A, Ferry L, Fajardy I, et al. (2012) Maternal diets trigger sex-specific divergent trajectories of gene expression and epigenetic systems in mouse placenta. PLOS ONE 7, e47986.

9. Du P, Kibbe WA \& Lin SM (2008) lumi: a pipeline for processing Illumina microarray. Bioinformatics 24, 1547-1548.

10. Wang X, Cheng M, Zhao M, et al. (2013) Differential effects of high-fat-diet rich in lard oil or soybean oil on osteopontin 
expression and inflammation of adipose tissue in diet-induced obese rats. Eur J Nutr 52, 1181-1189.

11. Cortez M, Carmo LS, Rogero MM, et al. (2013) A high-fat diet increases IL-1, IL-6, and TNF- $\alpha$ production by increasing $\mathrm{NF}-\kappa \mathrm{B}$ and attenuating PPAR- $\gamma$ expression in bone marrow mesenchymal stem cells. Inflammation 36, 379-386.

12. Gheorghe CP, Goyal R, Holweger JD, et al. (2009) Placental gene expression responses to maternal protein restriction in the mouse. Placenta 30, 411-417.

13. Krasnow SM, Nguyen MLT \& Marks DL (2011) Increased maternal fat consumption during pregnancy alters body composition in neonatal mice. Am J Physiol Endocrinol Metab 301, E1243E1253.

14. Kalaria RN, Maestre GE, Arizaga R, et al. (2008) Alzheimer's disease and vascular dementia in developing countries: prevalence, management, and risk factors. Lancet Neurol 7, 812-826.

15. Yang H-T, Sheen Y-J, Kao C-D, et al. (2013) Association between the characteristics of metabolic syndrome and Alzheimer's disease. Metab Brain Dis 28, 597-604.

16. Arroyo JA, Li C, Schlabritz-Loutsevitch N, et al. (2010) Increased placental XIAP and caspase 3 is associated with increased placental apoptosis in a baboon model of maternal nutrient reduction. $A m \mathrm{~J}$ Obstet Gynecol 203, 364.e313-364.e318.

17. Belkacemi L, Chen CH, Ross MG, et al. (2009) Increased placental apoptosis in maternal food restricted gestations: role of the Fas pathway. Placenta 30, 739-751.

18. Goksu Erol AY, Nazli M \& Yildiz SE (2012) Expression levels of cyclooxygenase-2, tumor necrosis factor- $\alpha$ and inducible NO synthase in placental tissue of normal and preeclamptic pregnancies. J Matern Fetal Neonatal Med 25, 826-830.

19. Johnson RD, Walsh SW, Everson WV, et al. (1995) Differentiation and growth on a fibrin matrix modulate the cyclooxygenase expression and thromboxane production by cultured human placental trophoblasts. Prostaglandins Leukot Essent Fatty Acids 52, 21-27.

20. Robinson JS, Redman CW, Clover L, et al. (1979) The concentrations of the prostaglandins $\mathrm{E}$ and F, 13 14-dihydro-15-oxoprostaglandin $\mathrm{F}$ and thromboxane B2. In tissues obtained from women with and without pre-eclampsia. Prostaglandins Med 3, 223-234.

21. Wetzka B, Nusing R, Charnock-Jones DS, et al. (1997) Cyclooxygenase- 1 and -2 in human placenta and placental bed after normal and pre-eclamptic pregnancies. Hum Reprod 12, 2313-2320.

22. Redman CW \& Sargent IL (2001) The pathogenesis of preeclampsia. Gynecol Obstet Fertil 29, 518-522.

23. Roberts JM, Balk JL, Bodnar LM, et al. (2003) Nutrient involvement in preeclampsia. J Nutr 133, 1684S-1692S.

24. Polydorides AD, Kalish RB, Witkin SS, et al. (2007) A fetal cyclooxygenase- 2 gene polymorphism is associated with placental malperfusion. Int J Gynecol Pathol 26, 284-290.

25. Varastehpour A, Radaelli T, Minium J, et al. (2006) Activation of phospholipase A2 is associated with generation of placental lipid signals and fetal obesity. J Clin Endocrinol Metab 91, 248-255.

26. Yang N, Higuchi O, Ohashi K, et al. (1998) Cofilin phosphorylation by LIM-kinase 1 and its role in Rac-mediated actin reorganization. Nature 393, 809-812.

27. Ritchey L, Ottman R, Roumanos M, et al. (2012) A functional cooperativity between Aurora A kinase and LIM kinase1: implication in the mitotic process. Cell Cycle 11, 296-309.

28. Cheng AK \& Robertson EJ (1995) The murine LIM-kinase gene (limk) encodes a novel serine threonine kinase expressed predominantly in trophoblast giant cells and the developing nervous system. Mech Dev 52, 187-197. 